\title{
Data on the quality of life after cytoreductive surgery and hyperthermic intraperitoneal chemotherapy for peritoneal malignancies: does it concern patients with gastric cancer?
}

\author{
Malgorzata Skierucha ${ }^{1,2 *}$, Karol Polom ${ }^{3}$, Karol Rawicz-Pruszynski ${ }^{1}$, Jerzy Mielko ${ }^{1}$, \\ Robert Sitarz ${ }^{1,2}$, Ryszard Maciejewski ${ }^{2}$, Franco Roviello ${ }^{3}$, Wojciech Polkowski ${ }^{1}$
}

${ }^{1}$ Department of Surgical Oncology, Medical University of Lublin, 20-081 Lublin, Poland

2 Department of Human Anatomy, Medical University of Lublin, 20-950 Lublin, Poland

${ }^{3}$ Department of Medicine, Surgery and Neurosciences, University of Siena, 53100 Siena, Italy

\section{ARTICLE INFO \\ Received 05 December 2017 Accepted 29 December 2017}

\section{Keywords:}

cytoreductive surgery (CRS), hyperthermic intraperitoneal chemotherapy (HIPEC), quality of life (QoL), gastric cancer (GC).

\section{ABSTRACT}

Introduction. So far there are no reports devoted exclusively to the quality of life after cytoreductive surgery and hyperthermic intraperitoneal chemotherapy in metastatic gastric cancer. Current literature concerning this issue was, thus, reviewed in order to: 1) search for such data concerning metastatic gastric cancer; 2 ) assess if the latest reviews evenly pertain to all peritoneal surface malignancies; and 3) conclude if they are a reliable source of data for patients with metastatic gastric cancer.

Materials and Methods. The electronic PubMed/MEDLINE and EMBASE databases were retrieved for studies concerning the influence of cytoreductive surgery and hyperthermic intraperitoneal chemotherapy on quality of life in patients with metastatic gastric cancer and regardless of initial diagnosis. The data on the number of patients with particular tumours were analysed and the results were presented in the form of a table.

Results. Approximately half of all patients encompassed by the reviews had a form of primary peritoneal surface malignancies. Within peritoneal metastases, the most numerous were colorectal (21-24\%) and ovarian cancers (5-15\%). Gastric cancers and sarcomas were the smallest defined subgroups ( $4 \%$ each).

Conclusions. The promising outcomes in quality of life after cytoreductive surgery and hyperthermic intraperitoneal chemotherapy in primary peritoneal surface malignancies might differ from rarely reported ones in metastatic gastric cancer. The problem needs further, gastric cancer-devoted investigations.
\end{abstract}

\section{INTRODUCTION}

Cytoreductive surgery (CRS) and hyperthermic intraperitoneal chemotherapy (HIPEC) have been a breakthrough in the management of peritoneal surface malignancies (PSM). These treatment modalities consist of highly extensive surgery followed by administration of cytotoxic drugs directly into the peritoneal cavity. The idea of the procedure is to remove all residual cancer cells which might be a future origin of relapse or new metastases. Additionally, intraperitoneal (IP) chemotherapy enables applying high doses of drugs while omitting the adverse effects of intravenous treatment which seems to have minimal effect due

\footnotetext{
${ }^{\star}$ Corresponding author

e-mail: malgorzata.skierucha@gmail.com
}

to the blood-peritoneal barrier and poor vascularisation of the tumour tissue [1-3].

The peritoneum might be affected by the neoplastic process either initially or as a destination of metastases from other intraabdominal cancers. Malignant peritoneal mesothelioma (MPM) and pesudomyxoma peritonei (PMP) are primary peritoneal surface malignancies (PSM), whereas secondary PSM comprise peritoneal metastases (PM) from different carcinomas, most frequently from colorectal, ovarian and gastric cancers (CRC, OC, GC). Sometimes, the term 'peritoneal carcinomatosis' (PC) is used to highlight the massive extent of the peritoneal seeding. However, this term should be abandoned as in fact it indicates multiple, diffuse end-stage of PM. 
Combined CRS and HIPEC, as an aggressive approach to PSM, generally contributes to overall survival [4]. However, the effects differ among the tumour types. The prognosis of patients with MPM and PMP after CRS and HIPEC has significantly improved, and this positive tendency is also observed in selected patients with limited PM. Nevertheless, the benefit comes at the expense of increased morbidity following such extensive surgeries. In experienced, tertiary centres, major postoperative morbidity rates reach $10-55 \%$, but mortality does not exceed $10 \%$ [5]. Therefore, the risk of postoperative complications and perioperative mortality following combined CRS and HIPEC is comparable to other major surgical procedures, such as oesophagectomy or pancreatoduodenectomy [6,7].

The potential benefits of CRS and HIPEC in PSM should be carefully confronted with the influence on the quality of life (QoL), which is a subjective and multidimensional parameter that encompasses physical and occupational function, psychological state, social interaction and somatic sensation [8]. The instruments used in QoL assessment are adapted to study the performance of patients on different daily planes. The most common are FACT-C, EORTC-QLQC30 or EORTC-QLQ-CR38, SF-36 and ECOG. Others, such as ADL, BPI, CES-D and LAS are less popular $[9,10]$.

So far there are no reports devoted exclusively to QoL after CRS and HIPEC in gastric PM, and only few papers discuss this subgroup separately. Nevertheless, it appears that since the first attempts of HIPEC in GC, median survival has not substantially changed. In one paper from 1991, the reported median survival was 14.6 months - and it was comparable to 14.3 months in a different paper from 2016 $[11,12]$.

The aim of the study was: 1) to search for data on QoL after CRS and HIPEC in metastatic GC; 2) to analyse the latest reviews about QoL after CRS and HIPEC from an epidemiological point of view, so as to assess if their results evenly pertain to all PSM; and 3) to conclude if they are a reliable source of data for patients with PM from GC.

\section{MATERIALS AND METHODS}

The electronic PubMed/MEDLINE and EMBASE databases were retrieved for studies about the influence of CRS and HIPEC on QoL in patients with metastatic GC. The search was conducted in December 2016 and involved terms: 'CRS' + 'HIPEC' + 'QoL' + 'metastatic gastric cancer'. There were no limitations regarding the type of the article, year of publication, text availability or language.

The consecutive search was aimed at the reviews that concern the influence of CRS and HIPEC on QoL regardless of initial diagnosis. The terms were 'CRS' + 'HIPEC'+ 'QoL'. The abstracts of 33 shortlisted papers were checked to decide if they were directly devoted to QoL after CRS and HIPEC, if they were not limited to only one specific tumour and if they based on data from primary studies. Finally, three reviews were found to meet all the conditions and were used in further analysis. These were: Lambert et al. [9], Seretis et al. [10] and Shan et al. [13].

The information about the quantity of subgroups with particular tumours in the original studies used in these three reviews has been assessed quantitatively in an Excel sheet. The data from 14 out of 15 original papers analysed by Shan et al. were registered, the remaining one did not provide the detailed number of subgroups. From Seretis et al. [10], the data from 18 out of 20 studies were used, and from Lambert et al. [9], we employed the data from 20 out of 22 original studies. In the both reviews, one remaining paper was inaccessible and the other did not provide the detailed number of subgroups (the same as in Shan et al. [13]).

In the case of only percentage data about the number of enrolled patients, the absolute value was calculated. In these cases, the calculated subgroups were controlled by adding them up. No discrepancy occurred. Due to the fact that certain patients were excluded from QoL analyses, the total number of patients in our analysis was, in some cases, not equal to the declared number of patients in the original studies.

All diagnoses mentioned in the original papers were included in the final sheet. These were: CRC, OC, GC, appendiceal cancer (AC); pseudomyxoma peritonei (PMP); primary peritoneal neoplasms (PPN), MPM, sarcoma, unknown, others (undefined by authors). Additionally, AP, PMP, PPN and MPM were summed up, as they are forms of primary PSM. All the data are presented in Table 1 and the details of the studies comprised in the analysed reviews are enclosed in the supplementary materials.

\section{RESULTS}

In our initial search aimed at QoL after CRS and HIPEC in $\mathrm{GC}$, the relevant information was found in 8 papers. Only one of these was devoted solely to $\mathrm{GC}$, but because of the lack of objective QoL data, it was rejected as being an insufficient source of information [14].

From the conducted epidemiological analysis, it appeared that the numerical details of the three analysed reviews were almost the same, as they were based on nearly the same primary studies. Approximately half of the patients encompassed by these reviews had a form of PSM, with an outstanding majority of AC. Within the subgroups of PM, the most numerous were CRCs, which summed up to approximately 22-24\%, whereas, OCs represented approximately $5-15 \%$ of all diagnoses. Sarcomas and GCs were the smallest defined subgroups, and these represented approximately $4 \%$ of all diagnoses.

\section{DISCUSSION}

In some subtypes of $\mathrm{GC}$, the most frequent site of metastases is the peritoneum [15]. The stage of disease with peritoneal seeding has fatal, maximal 3-month long prognosis $[16,17]$ and the standard palliative treatment is based on systemic chemotherapy. According to the REGATTA trial, primary gastrectomy added as the introduction to chemotherapy did not prolong the survival of patients with metastatic GC [18]. Subsequently, the recent GYMSSA trial compared the survival outcomes of D2 gastrectomy expanded by metastasectomy and systemic therapy with chemotherapy alone [19]. The REGATTA and GYMSSA trials, however, also differed in the characteristics of interventions, as the 
Table 1. The numerical and percentage division of patients enrolled for QoL analysis in studies comprised in the reviews of Shan et al. [13], Seretis et al. [10] and Lambert et al. [9]. The details of the primary studies are contained in the supplementary materials

\begin{tabular}{|c|c|c|c|c|c|c|c|c|c|c|}
\hline & & \multicolumn{3}{|c|}{${ }^{1}$ Shan et al. [13] } & \multicolumn{3}{|c|}{${ }^{2}$ Seretis et al. [10] } & \multicolumn{3}{|c|}{${ }^{3}$ Lambert et al. [9] } \\
\hline & & No. & $\%$ & Sum & No. & $\%$ & sum & No. & $\%$ & sum \\
\hline \multirow{3}{*}{$\begin{array}{l}\text { Peritoneal } \\
\text { metastases }\end{array}$} & CRC & 167 & $23.7 \%$ & \multirow{3}{*}{$33.5 \%$} & 243 & $22.4 \%$ & \multirow{3}{*}{$41.8 \%$} & 267 & $23.4 \%$ & \multirow{3}{*}{$43.3 \%$} \\
\hline & OC & 38 & $5.4 \%$ & & 167 & $15.4 \%$ & & 175 & $15.4 \%$ & \\
\hline & GC & 31 & $4.4 \%$ & & 43 & $4.0 \%$ & & 52 & $4.5 \%$ & \\
\hline \multirow{4}{*}{$\begin{array}{l}\text { Primary peritoneal } \\
\text { surface malignancies }\end{array}$} & AP & 294 & $41.6 \%$ & \multirow{4}{*}{$54.9 \%$} & 309 & $28.5 \%$ & \multirow{4}{*}{$49.6 \%$} & 311 & $27.3 \%$ & \multirow{4}{*}{$48.7 \%$} \\
\hline & PMP & 46 & $6.5 \%$ & & 149 & $13.8 \%$ & & 157 & $13.8 \%$ & \\
\hline & PPN & 1 & $0.1 \%$ & & 16 & $1.5 \%$ & & 18 & $1.6 \%$ & \\
\hline & MPM & 47 & $6.7 \%$ & & 65 & $6.0 \%$ & & 68 & $6.0 \%$ & \\
\hline \multirow{4}{*}{ Others } & sarcoma & 31 & $4.4 \%$ & \multirow{3}{*}{$11.6 \%$} & 31 & $2.9 \%$ & \multirow{3}{*}{$8.6 \%$} & 31 & $2.7 \%$ & \multirow{3}{*}{$8.0 \%$} \\
\hline & unknown & 20 & $2.8 \%$ & & 19 & $1.8 \%$ & & 19 & $1.7 \%$ & \\
\hline & others & 31 & $4.4 \%$ & & 41 & $3.9 \%$ & & 41 & $3.6 \%$ & \\
\hline & ${ }^{4}$ Total & 706 & $100 \%$ & & 1183 & $100 \%$ & & 1139 & $100 \%$ & \\
\hline
\end{tabular}

$\mathrm{CRC}=$ colorectal cancer; $\mathrm{OC}=$ ovarian cancer; $\mathrm{GC}=$ gastric cancer; $\mathrm{AC}=$ appendiceal cancer; $\mathrm{PMP}=$ pseudomyxoma peritonei; PPN = primary peritoneal neoplasms, MPM = malignant peritoneal mesothelioma

${ }^{1}$ Shan et al. [20]- data from 14 out of 15 studies analysed in the review, the remaining one did not provide the detailed number of the subgroups

${ }^{2}$ Seretis et al. [17] - data from 18 out of 20 studies analysed in the review, one remaining paper was inaccessible to the authors and the other did not provide the detailed number of subgroups

${ }^{3}$ Lambert et al. [16]- data from 20 out of 22 studies analysed in the review, one remaining paper was inaccessible to the authors and the other did not provide the detailed number of subgroups

${ }^{4}$ The total number did not equal the declared number of patients in all studies, because in some studies, certain patients were excluded from QoL analyses.

In one study, the declared number of patients was smaller than the sum of subgroups

former involved limited, palliative, D1 gastrectomy. According to the recently published FLOT3 trial, it is significant to define the stage and resectability of metastatic disease, as patients with oligometastatic GCs treated with neoadjuvant chemotherapy followed by $\mathrm{R} 0$ resection showed favourable survival, when compared to patients treated only by palliative means [20].

Extensive surgery, additionally prolonged by IP chemotherapy, is a physical and mental challenge for a fragile oncologic patient. The severity of the procedure is expressed in mortality and morbidity rates which are mostly responsible for deterioration in the postoperative QoL and are similar in all cancers. The postoperative malaise is acceptable, as it is only temporary. What seems to be more important is a lasting decline in physical, mental and social activities, which might often be worse than a shorter but more satisfactory life. Moreover, it is not without significance that life expectations vary according to the realistic prospects of survival and to the performance status at the starting point. Therefore, the issue of QoL after CRS and HIPEC cannot be neglected and should be analysed separately in different PSMs.

In studies devoted to PSM from any origin, CRS and HIPEC were shown not to have negative long-term impact on the QoL of surviving patients [9] and to have general small/medium benefits in a one year perspective [13]. Overall, QoL returns or exceeds the preoperative level within the first year after procedures and achieves the peak point at 18 months [10-13,21]. Nevertheless, such conclusions come from the studies where QoL after CRS and HIPEC was analysed regardless of the tumours origin. In our analysis, more than $70 \%$ of all diagnoses were either some PSM or CRC. Generally, the patients with PMP/AC have very good baseline performance status, which was clearly shown in the report where $84 \%$ of patients who started treatment had an ECOG score 0 [22]. Their QoL returned or exceeded the preoperative level within 6 months [23]. Among the patients with colon PM, QoL returned to the baseline after 6 months [24].

The doubt if the general data on QoL after CRS and HIPEC reliably present the situation of patients with peritoneal dissemination of GC comes from the observation that in overall analyses, the cases of upper gastrointestinal malignancies are in minority. The trends in clinical outcomes in such neoplasms remain dramatically poor, even despite the recent advances in the multimodality treatment (CROSS trial, ESPAC-3 trial) [6,7]. Indeed, QoL after operations performed for oesophageal or pancreatic head carcinomas was reported to be dramatically impaired and never returned to preoperative values [25-26]. Giving that GC is a typical upper gastrointestinal malignancy and that CRS and HIPEC in GC patients is more fatal, as mortality rates even reach $8 \%$ [17], there is a logical question about QoL after CRS and HIPEC in GC. The issue requires further investigation.

\section{CONCLUSIONS}

The influence of CRS and HIPEC on QoL depends largely on the origin and histology of the tumour and the range of PSM. From our statistics, it appeared that gastric PM constituted only about $4 \%$ of all diagnoses comprised in the big reviews devoted to QoL after CRS and HIPEC (Table 1).

Giving the dramatically different prognoses in PSM and gastric PM, the tendency towards generalisation might cause misleading interpretations [4]. The promising outcomes in the QoL after CRS and HIPEC in the numerically superior cases of PSM cover miserable and rarely reported ones in gastric PM. To assess the value of the procedure in this case, separate studies need to be performed. 


\section{CONFLICT OF INTEREST}

The authors declare that they have no conflict of interest. No funding or financial assistance was received by the authors.

\section{REFERENCES}

1. Yonemura Y, Endou Y, Sasaki T, Hirano M, Mizumoto A, Matsuda T, et al. Surgical treatment for peritoneal carcinomatosis from gastric cancer. Eur J Surg Oncol 2010;36:1131-1138.

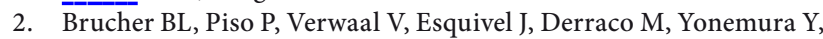
et al. Peritoneal carcinomatosis: cytoreductive surgery and HIPECoverview and basics. Cancer Invest 2012;30:209-224.

3. Cotte E, Colomban O, Guitton J, Tranchand B, Bakrin N, Gilly FN, et al. Population pharmacokinetics and pharmacodynamics of cisplatinum during hyperthermic intraperitoneal chemotherapy using a closed abdominal procedure. J Clin Pharmacol 2011;51:9-18.

4. Desantis M, Bernard JL, Casanova V, Cegarra-Escolano M, Benizri E, Rahili AM, et al. Morbidity, mortality, and oncological outcomes of 401 consecutive cytoreductive procedures with hyperthermic intraperitoneal chemotherapy (HIPEC). Langenbecks Arch Surg 2015;400:37-48

5. Desiderio J, Chao J, Melstrom L, Warner S, Tozzi F, Fong Y, et al. The 30-year experience-A meta-analysis of randomised and highquality non-randomised studies of hyperthermic intraperitoneal chemotherapy in the treatment of gastric cancer. Eur J Cancer 2017;79:1-14.

6. Shapiro J, van Lanschot JJ, Hulshof MC, van Hagen P, van Berge Henegouwen MI, Wijnhoven BP, et al. Neoadjuvant chemoradiotherapy plus surgery versus surgery alone for oesophageal or junctional cancer (CROSS): long-term results of a randomised controlled trial. Lancet Oncol 2016;16:1090-1098.

7. Neoptolemos JP, Moore MJ, Cox TF, Valle JW, Palmer DH, McDonald AC, et al. Effect of adjuvant chemotherapy with fluorouracil plus folinic acid or gemcitabine vs observation on survival in patients with resected periampullary adenocarcinoma: the ESPAC-3 periampullary cancer randomized trial. JAMA 2012;308:147-156.

8. Schipper H, Clinch JJ, Olweny CLM.: Quality of life studies: Definitions and conceptual issues; in Spilker B (ed): Quality of Life and Pharmacoeconomics in Clinical Trials. Philadelphia: LippincottRaven Publishers; 1996:11-23.

9. Lambert LA, Harris A. Palliative cytoreductive surgery and hyperthermic intraperitoneal chemoperfusion : current clinical practice or misnomer? J Gastrointest Oncol 2016;7:112-121.

10. Seretis C, Youssef $\mathrm{H}$. Quality of life after cytoreductive surgery and intraoperative hyperthermic intraperitoneal chemotherapy for peritoneal surface malignancies: A systematic review: Eur J Surg Oncol 2014;40:1605-1613.

11. Yonemura Y, Fujimura T, Fushida S, Takegawa S, Kamata T, Katayama K, et al. Hyperthermo-chemotherapy combined with cytoreductive surgery for the treatment of gastric cancer with peritoneal dissemination. World J Surg 1991;15:530-536.

12. Wu HT, Peng KW, Ji ZH, Sun JH, Zhang Q. Yang XJ, et al. Cytoreductive surgery plus hyperthermic intraperitoneal chemotherapy to treat gastric cancer with ascites and/or peritoneal carcinomatosis: Results from a Chinese center: Eur J Surg Oncol 2016;42:1024-1034.
13. Shan LL, Saxena A, Shan BL, Morris DL. Quality of life after cytoreductive surgery and hyperthermic intraperitoneal chemotherapy for peritoneal carcinomatosis: A systematic review and meta-analysis: Surg Oncol 2014;23:199-210.

14. Wu HT, Peng KW, Ji ZH, Sun JH, Zhang Q, Yang XJ, et al. Cytoreductive surgery plus hyperthermic intraperitoneal chemotherapy with lobaplatin and docetaxel to treat synchronous peritoneal carcinomatosis from gastric cancer: Results from a Chinese center. Eur J Surg Oncol 2016;42:1024-34

15. Riihimäki M, Hemminki A, Sundquist K, Sundquist J, Hemminki K. Metastatic spread in patients with gastric cancer. Oncotarget 2016;7:52307-52316.

16. Yonemura Y, Canbay E, Li Y, Coccolini F, Glehen O, Sugarbaker PH, et al. A comprehensive treatment for peritoneal metastases from gastric cancer with curative intent. Eur J Surg Oncol 2016;42:1123-31.

17. Chia CS, You B, Decullier E, Vaudoyer D, Lorimier G, Abboud $\mathrm{K}$, et al. Patients with Peritoneal Carcinomatosis from Gastric Cancer Treated with Cytoreductive Surgery and Hyperthermic Intraperitoneal Chemotherapy: Is Cure a Possibility? Ann Surg Oncol 2016;23:1971-1979.

18. Fujitani K, Yang HK, Mizusawa J, Kim YW, Terashima M, Han SU, et al. Gastrectomy plus chemotherapy versus chemotherapy alone for advanced gastric cancer with a single non-curable factor (REGATTA): a phase 3, randomised controlled trial. Lancet Oncol 2016;17:309-318.

19. Kerkar SP, Kemp CD, Duffy A, Kammula US, Schrump DS, Kwong $\mathrm{KF}$, et al. The GYMSSA trial: a prospective randomized trial comparing gastrectomy, metastasectomy plus systemic therapy versus systemic therapy alone. Trials 2009;10:121.

20. Al-Batran SE, Homann N, Pauligk C, Illerhaus G, Martens UM, Stoehlmacher J, et al. Effect of Neoadjuvant Chemotherapy Followed by Surgical Resection on Survival in Patients With Limited Metastatic Gastric or Gastroesophageal Junction Cancer: The AIOFLOT3 Trial. JAMA Oncol 2017 [unpublished data].

21. Albertsmeier M, Hauer A, Niess H, Werner J, Graeb C, Angele MK. Quality of life in peritoneal carcinomatosis: A prospective study in patients undergoing cytoreductive surgery and hyperthermic intraperitoneal chemotherapy (HIPEC). Dig Surg 2014;31:334-340.

22. Baratti D, Kusamura S, Cabras AD, Bertulli R, Hutanu I, Deraco M. Diffuse malignant peritoneal mesothelioma: Longterm survival with complete cytoreductive surgery followed by hyperthermic intraperitoneal chemotherapy (HIPEC). Eur J Cancer 2013;49:3140-3148.

23. Jess P, Iversen LH, Nielsen MB, Hansen F, Laurberg S, Rasmussen PC. Quality of life after cytoreductive surgery plus early intraperitoneal postoperative chemotherapy for pseudomyxoma peritonei: a prospective study. Dis Colon Rectum 2008;51:868-874.

24. Chia CS, Tan GH, Lim C, Soo KC, Teo MC. Prospective Quality of Life Study for Colorectal Cancer Patients with Peritoneal Carcinomatosis Undergoing Cytoreductive Surgery and Hyperthermic Intraperitoneal Chemotherapy. Ann Surg Oncol 2016;23:2905-13.

25. Eaton AA, Gonen M, Karanicolas P, Jarnagin WR, D'Angelica MI, DeMatteo R, et al. Health-Related Quality of Life After Pancreatectomy: Results From a Randomized Controlled Trial. Ann Surg Oncol 2016;23:2137-2145.

26. Malmström M, Klefsgard R, Ivarsson B, Roman M, Johansson J. Quality of life measurements as an indicator for timing of support after oesophagectomy for cancer: a prospective study. BMC Health Serv Res 2015;15:96. 